\title{
Diagnostic Value of Dental Pulp Stones in the Early Diagnosis of Ischemic Heart Diseases
}

\author{
Fatemeh Ezoddini-Ardakani ${ }^{1}$, Seyedeh Mahdieh Nemayandeh ${ }^{2 *}$, \\ Seyed Mahmood Sadrbafghi ${ }^{2}$, Sedigheh Hajihashemi², Mahmood Emami², \\ Forouzandeh Ghasemi Kahtouei ${ }^{2}$, Leila Hadiani' ${ }^{2}$, Mohammad Hossein Ahmadieh ${ }^{2}$, \\ Maliheh Moeini' ${ }^{1}$, Seyed Hossein Razavi ${ }^{1}$, Sajad Besharati ${ }^{3}$ \\ ${ }^{1}$ Oral and Maxillofacial Radiology Department, Faculty of Dentistry, Shahid Sadoughi University of Medical \\ Sciences, Yazd, Iran \\ ${ }^{2}$ Yazd Cardiovascular Research Center, Shahid Sadoughi University of Medical Sciences, Yazd, Iran \\ ${ }^{3}$ Shahid Beheshti University of Medical Sciences, Tehran, Iran \\ Email: "drnamayandeh@gmail.com
}

Received 30 December 2014; accepted 7 March 2015; published 16 March 2015

Copyright (C) 2015 by authors and Scientific Research Publishing Inc.

This work is licensed under the Creative Commons Attribution International License (CC BY).

http://creativecommons.org/licenses/by/4.0/

(c) (i) Open Access

\begin{abstract}
Background: Pulp stones are calcified masses formed in the primary and permanent dentitions. The ischemic cardiovascular disorders (CVD) can be the first health problem of the world. It seems that there is a relationship between pulp stones and cardiovascular diseases to determine the diagnostic value of panoramic dental radiographs as non-invasive test for the early detection of CVD. Methods and Materials: The subjects of the study came from patients presenting to Dental Radiology Department of Dental School in Yazd who aged 30 - 64 years, had 8 natural teeth. They were referred to the Cardiovascular Center of Afshar Hospital in Yazd for cardiovascular evaluation. To set the cut-off point, the ratio of teeth with pulp stones to the total number of teeth for each person was calculated and also ABI for diagnosing IHD via ROC curve was used. Results: Only $3.8 \%$ of patients without pulp stone were affected by ischemic heart disease (IHD). About Sixty seven percent (67.3\%) of individuals had at least one tooth with pulp stone. In individuals without IHD, $5 \%$ of the teeth showed pulp stone while this rate was $45 \%$ (9 times) in CVD patients. The number of patients for whom the ratio of teeth with pulp stone to total number of teeth was 0.2 or more, was 138.7 times greater than the other. Conclusion: The dentists, who order panoramic radiographs for the treatment of patients, exactly study those teeth for the presence of pulp stones. The ratio of teeth with pulp stone to total teeth equal 0.2 or more was a good tool for early detection of CVD.
\end{abstract}

"Corresponding author.

How to cite this paper: Ezoddini-Ardakani, F., Nemayandeh, S.M., Sadrbafghi, S.M., Hajihashemi, S., Emami, M., Kahtouei, F.G., Hadiani, L., Ahmadieh, M.H., Moeini, M., Razavi, S.H. and Besharati, S. (2015) Diagnostic Value of Dental Pulp Stones in the Early Diagnosis of Ischemic Heart Diseases. Health, 7, 336-345. http://dx.doi.org/10.4236/health.2015.73038 


\section{Keywords}

\section{Pulp Stone, Cardiovascular Disease, Panoramic Radiography}

\section{Introduction}

Cardiovascular diseases are among the leading causes of mortality in the USA and also in our own society. According to the findings of GBD (Global Burden of Disease), it is expected that the mortality rate of non-contagious diseases will rise with a $77 \%$ from 28.1 million to 94.7 million per year by 2020 . Also, the ischemic diseases of the heart which ranked as the fifth cause of mortality in 1990 will be the first cause of mortality in 2020. This shows that the ischemic cardiovascular disorders can be the first health problem of the world, so demanding a comprehensive revision of the preventive and treatment programs [1].

Pulp stones are calcified masses formed in the primary and permanent dentitions. Many causes have been considered for these stones including nanobacteria, powerful occlusal forces, etc. [2]. It has been reported that osteopontin is a new constituent of atherosclerotic plaques playing a role in plaque calcification. It has also been found that osteopontin produced by macrophages plays a basic role in the production of calcification centers within the necrotic areas of breast cancer [3]. Similar formation of these calcified stones has been observed in other regions of the body as carotid and renal arteries [2].

It seems that these isolated calcified masses are more commonly seen in mature teeth though the incidence of pulp stones increases with age. However, these stones have also been observed in normal young teeth with healthy pulps [3].

Kjander E.O. and Ciftcioglu of the Biochemistry Department of Kuepio University in Finland studied all the developmental phases of nanobacteria and found that nanobacteria could produce the biologic apatite on their own cellular covering. These mineral substances are almost similar to the calcified tissues and renal calculi. The purpose of their study was to investigate the role of nanobacteria and their activities in the calcification of atherosclotic plaque, renal calculi, and pulpal calcifications. In fact, nanobacteria are an active center for the formation of calcified minerals [6]. So there may be a common cause for both pulp stones and atheromatous plaques.

Arteriosclerosis is the most common cause of IHD including angina pectoris, myocardial infarction, and also cerebrovascular diseases as stroke and peripheral artery diseases [4]. An investigation of post mortal autopsies of patients in the USA indicated that between the ages of 30 - 39 years, less than $20 \%$ of the patients had obstruction of one or more coronary arteries by more than $50 \%$ [5]. Furthermore, over than $36 \%$ of mortality in the towns of Yazd province in Iran is due to cardiovascular diseases. The statistics of risk factors in Yazdare as the following: hypertension $25 \%$, blood lipid disturbance $58 \%$, cholesterol more than $240,12 \%$, known diabetes $11 \%$, Impaired glucose tolerance test (IGTT) $8.5 \%$, overweight $35 \%$, obesity $16 \%$, and smoking $13 \%$ (24\% males and $0.5 \%$ females) [1]. The diagnostic methods of ischemic cardiac disorders are electrocardiography or ECG (EKG), exercise test, echocardiography and angiography if necessary [4].

The study by Alison MA and co-workers aimed at determining the association between IMT (intimal medial thickness) and atherosclerotic calcification of this artery. It was stated that carotid IMT can be a measure of subclinical atherosclerosis and may predict the future cardiovascular accidents. Individuals with any type of carotid calcification have significantly greater thickness of intimal medial layer [7]. Therefore, finding a method for early diagnosis of IHD before development of clinical disease is of importance. Some studies have shown an association between the formation of pulp stones and atheromatous plaques in the vessels [8]-[11].

Nayak et al. found that the frequency of pulpal calcification is higher in subjects suffering from coronary atherosclerosis by radiographic examination [8]. Edds A.C. et al. studied the correlation between pulp stones and cardiovascular disease. They asserted that pulp calcification may have a pathogenesis similar to that of calcified vascular masses. This can lead to the popular use of dental radiography as a screening method for finding those who have a potential for cardiovascular diseases. The periapical radiographs of the patients were surveyed for the presence of pulp stones. 74\% (14/19) of the patients with IHD showed distinctive pulpal calcifications while only 39\% of the patients without a history of IHD had pulp stones. The findings indicated that the dental radiographs used to determine the presence or absence of pulpal calcifications may be used for screening IHD [9]. 
Maranhao de Moura et al. also found an increased frequency of pulp stones in people suffering from coronary atherosclerosis [10]. This association was observed in another study conducted by Khojastepour et al. as well [11]. They suggested that panoramic radiography can be used as a screening method for early detection of subjects who are at risk of ischemic heart diseases [11].

The study by Thanakun S. and Pornprasertsuk-Damrongsri investigated the prevalence of carotid artery calcification in panoramic X-rays of a group of Thai patients. Although the prevalence of carotid artery calcification (CAC) is not common in the Thai population, dentists should be aware of this calcification in the usual panoramic radiographs and refer the patient for cardiovascular and cerebral assessment [12].

In the study by Zeng J.F. et al., they isolated some bacteria from the pulp stones and cultivated them. These bacteria isolated from pulp stones were similar to nanobacteria regarding growth rate, form, and staining details. This unusual characteristic of bacteria may play an important role in the formation of pulpal calcifications [13]. The purpose of the study above was to determine the diagnostic value of pulp stones in prognosis and diagnosis of IHDs.

\section{Materials and Methods}

This test study evaluated diagnostic value of pulp stone as a noninvasive test for IHD detection. The subjects of the study came from patients presenting to Dental Radiology Department of Dental School in Yazd to take a dental radiograph since the winter of 2009 until the satisfactory number of samples were obtained. To determine the sample status regarding cardiovascular disorders, they were referred to the Yazd Cardiovascular research Center, Afshar Hospital in Yazd and the rest of the information in the questionnaire was obtained there (Appendix). The subjects that were include aged 30 - 64 years, had 8 natural teeth, and had panoramic dental radiographs for dental treatment. Exclusion criteria included:

Patients with contraindications of dental panoramic radiographs, those who were not inclined to participate after explaining the objectives of the study, and those who did not have enough time for the follow-up of cardiovascular disease were excluded from the study. Considering the inclusion and exclusion criteria, totally 200 subjects were selected from consecutive patients referred to dental radiography ward (100 with and 100 without pulp stone) for taking a dental radiograph. This study used the quantitative variables of age, height, weight, ABI systolic pressure, number of teeth and number of teeth with pulp stones and the qualitative variables of gender, presence of hypertension, dyslipidemia, diabetes, and smoking as the independent variables. It also used the qualitative variables of presence of IHD as the dependent variable. After sampling the qualified patients, a predetermined questionnaire including the patient's age, gender, name, and address was completed. Then, a panoramic radiograph was taken of each patient and interpreted by a dental radiologist. The total number of teeth and the number of teeth with pulp stones was recorded in the questionnaire.

If the first radiologist was doubtful about the presence of pulpal calcifications, then a second radiologist was called on to do the interpretation and diagnosis. The questionnaires were confidentially kept in the Radiology Department of Dental School and an introduction letter was given to the subject who was referred to the Cardiovascular Research Center of Afshar Hospital with another specific questionnaire. There, some expert questions were asked of the patients and the patients were examined for hypertension, diabetes, smoking, and dyslipidemia. Then, variables of, height, weight, and ankle and brachial systolic pressure were measured and recorded in the questionnaire. Electrocardiograms were prepared for all the subjects and Rose Chest Pain questionnaire and exercise tests were performed for all of them. In the case that angiography was indicated for a patient due to positive exercise test or other symptoms and signs, it was explained to the patient, the informed written consent was obtained, angiography was performed, and the results of the examinations, observations, and the general findings were recorded in the sent questionnaire showing whether the patient is affected by IHD or not. It should be pointed out that the physicians at the Cardiovascular Research Center were not aware of the presence or absence of pulp stones in patients. To analyze the data, SPSS17 was used and the statistical tests were applied to determine the cut-off point of the ratio of teeth with pulp stones to the total number of teeth. Also, ABI cut point was used to diagnose IHD via Roc Curve. P-value was set at $\leq 0.05$ and the area under the curve $>0.75$ in Roc Curve was set as the minimal acceptable surface. All the patients' private information were kept confidentially and all the costs and expenses of clinical and paraclinical examinations and tests were afforded by the researcher. No extra charges were imposed on patients. Informed written consent was obtained of each patient and if the patient was not inclined for participation, s/he was substituted with another one. This study was approved by the deputy 
in research and technology of Ministry of Health and Medical Education. Ethical approval was granted by the Committee of Research Ethics at Yazd Shahid Sadoughi University of Medical sciences.

\section{Results}

A total of 107 patients presenting to dental radiology of Dental School of Yazd for panoramic radiography were enrolled to in this research. Fifty subjects (46.7\%) weremen and 57 of them (53.3\%) were women. Their mean age was $46.09 \pm 7.88$ with an age range of $30-64$ years. The mean of the total number of their teeth was $24.14 \pm$ 3.65 with a range of 14 - 31 (Table 1). The mean of the number of teeth with pulp stones was $4.45 \pm 5.39$ with a range of $0-16$. The mean of ankles systolic pressure was $145.6 \pm 16.5$ with a range of $108-190 \mathrm{mmHg}$. The mean of brachial systolic pressure was $132.7 \pm 14.1$ with a range of $102-174 \mathrm{mmHg}$. On the whole, 68 patients (63.6\%) were healthy regarding IHD and the rest, i.e., 39 patients (36.4\%) were affected by cardiovascular disease. $20.6 \%$ of the subjects were affected by hypertension, $15 \%$ with diabetes, and $22.4 \%$ with dyslipidemia. Table 2 shows the frequency distribution of cardiovascular disease in terms of the presence of tooth with pulp stones.

The smoking rate in males was $12 \%$ and no case of smoking was observed in females. Since the number of teeth in the studied samples was not the same and ranged from 14 to 31, the teeth with dental pulp stones could not be used as a reference point and decisions could not be made on this basis. So, the ratio of teeth with pulp stones to the total number of teeth was calculated for each patient and used as a standard against which the diagnosis of IHD could be made (Table 3).

Based on the ROC curve and using the cut-off points table, the best cut-off point was 0.1952. At this cut-off point, test sensitivity was $94.9 \%$ and the specificity was $88.2 \%$ which are acceptable indices. Rounding the above-mentioned cut-off point (so that it does not reach the next cut-off point), 0.2 will be reached which is accidentally the mean of this index.

The best cut-off point obtained from this index is three teeth with pulp stones at which the rate of sensitivity is $94.9 \%$ and the specificity is $82.4 \%$. Another index used in this study to predict the IHD was the Ankle Brachial Index (ABI). Table 4 presents the mean of ABI of the samples in terms of description of ICD. Frequency distribution of results of the diagnosis of ICD using dental pulp stone in terms of disease status is presented in Table 5, and validity and accuracy of dental pulp stone ratio in diagnosis of CVD based on history of diabetes and hypertension is shown in Table 6.

Table 1. Demographic information of the subjects.

\begin{tabular}{ccccc}
\hline Variable & Mean & SD & Min & Max \\
\hline Age (yr.) & 46.1 & 7.9 & 30 & 64 \\
Height (cm) & 163.4 & 8.9 & 141 & 182 \\
Weight $(\mathrm{Kg})$ & 75.1 & 10.5 & 47 & 96 \\
BMI $\left(\mathrm{Kg} / \mathrm{m}^{2}\right)$ & 28.2 & 3.7 & 14.1 & 37.5 \\
Present teeth & 24.1 & 3.6 & 0 & 31 \\
Teeth with pulp stone & 4.5 & 5.4 & & 16 \\
\hline
\end{tabular}

Table 2. Frequency distribution of cardiovascular disease in terms of the presence of tooth with pulp stones.

\begin{tabular}{cccccccc}
\hline & \multicolumn{3}{c}{ Cardiovascular disease } \\
Status of pulp stone of tooth & \multicolumn{3}{c}{ Absent } & \multicolumn{2}{c}{ Present } & \multicolumn{2}{c}{ Total } \\
\cline { 2 - 8 } & Number & Percent & Number & Percent & Number & Percent \\
\cline { 2 - 7 } Without pulp stone & 50 & 96.2 & 2 & 3.8 & 52 & 100 \\
At least one tooth with pulp stone & 18 & 32.7 & 37 & 67.3 & 55 & 100 \\
Total & 68 & 63.6 & 39 & 36.4 & 107 & 100 \\
\hline
\end{tabular}

P-value $<0.000$; Patients with at least one tooth affected by pulp stones were significantly more affected by ischemic cardiovascular disease so that their risk of being affected by heart disease was 51.4 times $(\mathrm{OR}=51.4)$ greater than others. 
Table 3. Mean of ratio of teeth with pulp stones to total number of teeth in terms of Ischemic cardiovascular disorder (ICD) in the studied patients.

\begin{tabular}{cccccc}
\hline \multirow{2}{*}{ ICD } & $\mathrm{N}$ & \multicolumn{3}{c}{ Teeth with pulp stones to total number of teeth } \\
\cline { 3 - 6 } & & Mean & SD & Minimum & Maximum \\
\hline Absent & 68 & 0.05 & 0.1 & 0 & 0.6 \\
Present & 39 & 0.45 & 0.19 & 0 & 0.8 \\
Total & 107 & 0.2 & 0.24 & 0 & 0.8 \\
\hline
\end{tabular}

P-value $=0.000$; The surface beneath the curve (Roc) equaled $0.95 \pm 0.026$ (Mean \pm SE) with a probability value of $95 \%$ (CI95\%) ranging from 0.897 to 1 indicating the predictive power of this index (ratio of teeth with pulp stones to the total number of teeth) with a maximal error of $5 \%$.

Table 4. Mean of ankle brachial index (ABI) of the samples in the study in terms of description of ischemic cardiovascular disease (ICD).

\begin{tabular}{cccccc}
\hline \multirow{2}{*}{ ICD } & $\mathrm{N}$ & \multicolumn{3}{c}{ ABI ratio } \\
\cline { 3 - 6 } & 68 & Mean & SD & Minimum & Maximum \\
\hline Absent & 39 & 1.1 & 0.085 & 0.8 & 1.31 \\
Present & 107 & 1.09 & 0.068 & 0.94 & 1.27 \\
Total & 1.1 & 0.079 & 0.8 & 1.31 \\
\hline
\end{tabular}

P-value $=0.588$; The surface beneath the ROC for this index was $0.464 \pm 0.057$ with a probability limit of $95 \%$ from 0.353 to 0.575 . The difference with 0.5 was not significant at P-value $=0.539$.

Table 5. Frequency distribution of results of the diagnosis of ischemic cardiovascular disease (ICD) using dental pulp stone in terms of disease status (Gold Standard).

\begin{tabular}{|c|c|c|c|c|c|c|}
\hline \multirow{3}{*}{$\begin{array}{l}\text { Diagnosis of ICD using pulp } \\
\text { stone of tooth }\end{array}$} & \multicolumn{6}{|c|}{ Cardiovascular disease } \\
\hline & \multicolumn{2}{|c|}{ Absent } & \multicolumn{2}{|c|}{ Present } & \multicolumn{2}{|c|}{ Total } \\
\hline & Number & Percent & Number & Percent & Number & Percent \\
\hline Present & 37 & 94.9 & 8 & 5.1 & 45 & 42.1 \\
\hline Absent & 2 & 11.8 & 60 & 88.2 & 62 & 57.9 \\
\hline Total & 39 & 36.4 & 68 & 63.6 & 107 & 100 \\
\hline
\end{tabular}

P-value $=0.000$; Using the optimal cut-off point obtained from ROC $(0.2)$ for the number of teeth with pulp stone to the total number of teeth of each sample, the presence of IHD was diagnosed so that if the ratio of teeth with pulp stone to the total number of teeth was less than 0.2, then the patient was diagnosed not to have IHD and if it was greater than 0.2 , the presence of IHD was approved. The findings of this index compared to the patient's real status regarding IHD arrived at by clinical and paraclinical examinations and tests are presented in the table above. The diagnostic validity indices using pulp stone were calculated as the following: Sen $=94.9 \%$, Spe $=88.2 \%, \mathrm{PPV}=88.2 \%, \mathrm{NPV}=96.8 \%, \mathrm{Accuracy}=90.6 \%$.

Table 6. Validity and accuracy of dental pulp stone ratio in diagnosis of CVD based on history of diabetes and hypertension.

\begin{tabular}{ccccc}
\hline & Diabetics & Non-diabetics & Hypertensives & Normotensives \\
\hline Sensitivity & 88.9 & 96.7 & 100 & 92.6 \\
Specificity & 85.7 & 88.5 & 90 & 87.9 \\
PPV $^{*}$ & 88.9 & 80.6 & 92.3 & 100 \\
NPV $^{*}$ & 85.7 & 98.2 & 96.1 & 96.2 \\
Accuracy & 87.5 & 91.2 & 99.4 & 8 \\
\hline
\end{tabular}

*PPV: Positive Predictive Value, NPV: Negative Predictive Value.

\section{Discussion}

A total of 107 patients presenting to the dental radiology of Dental School of Yazd for panoramic radiographs were studied in this research. 50 of the subjects (46.7\%) were male and 57 of them (53.3\%) were female. Their mean age was $46.09 \pm 7.88$ with an age range of 30 - 64 years. The mean of the total number of their teeth was $24.14 \pm 3.65$ with a range of $14-31$. The mean of the number of teeth with pulp stones was $4.45 \pm 5.39$ with a range of $0-16$. Generally speaking, controversial debates have been raised on pulp calcifications and systemic disease. For example, Tames A. tried to find a correlation between pulp stones and various systemic diseases as cholelithiasis, renal calculi, gout, hypercemantosis, migraine, and Paget disease. Their data revealed the point that there was no definite correlation between these conditions and pulp calcifications. Yet, they found a strong 
correlation between calculi and the presence of atherosclerosis, osteitis deformans, and acromegaly [14]. In the study by Edds et al. (2005), 74\% (14/19) of the patients with IHD had pulpal calcifications while only 39\% $(14 / 36)$ of the patients without IHD had such stones. The study demonstrated that pulp stones occurred more in patients with IHD compared to those without it. In the present study, pulp stones were seen in $73.8 \%$ of the patients with IHD and in $26.2 \%$ of the patients without a IHD history. This is within the limits of the findings of the previous studies. Regarding the similarity of these two studies, there is the probability of a correlation between pulp stones and IHD [9]. The present study just focused on the medical history of the patients regarding cardiovascular diseases and there was a statistically significant correlation between the prevalence of pulpal calcifications and IHD. The correlation between cardiovascular diseases and pulp stones was studied by Ezoddini et al. (2011). Panoramic radiographs were taken of 61 patients aged 20 - 55 years presenting to the Cardiovascular Research Center of Afshar Hospital in Yazd for angiography. 82\% (31/38) of the patients with at least one obstructed vessel and 48\% (11/23) of the patients with normal angiography showed pulp stones. There was a statistically significant correlation between coronary artery stenosis and the presence of pulp stones. In conclusion, it was asserted that dental radiographs can be used as a quick screening method for the early diagnosis of coronary artery stenosis [15]. Horsley et al. conducted a study entitled: "Prevalence of Pulpal Calcifications and Carotid Artery Using Digital Radiographs”. The digital panoramic radiographs of the patients were studied in the dental oncology clinic if the patients' carotid artery bifurcation was visible, and had no restored molar or canine teeth. One endodontist studied the pulpal calcifications and one oral radiologist studied the bifurcation calcifications in the same radiographs. The presence of pulp stone was assessed as evidence of carotid calcification screening test. There was a significantly high prevalence of pulp and carotid calcification in patients aged 60+ compared to the young patients. The efficiency of pulp calcification in carotid calcification screening was $66.4 \%$. They concluded that these two simultaneously calcified regions are seen in older patients. Also, the presence of pulp calcification is not a powerful predictor of the presence of carotid calcification [16]. Kansu et al. studied 60 nephrology patients (29 hemodialysis and 31 kidney graft). They wanted to know if dental calcification could be a diagnostic marker of carotid artery calcification in patients with renal disease. Panoramic and periapical radiographs were recorded twice for all the patients by three interpreters to determine the presence or absence of narrowing of pulpal chamber and pulp stone in the pulp chamber and canal. Ultrasonography approved carotid calcifications findings of panoramic radiography. Also, there was no significant correlation between pulp stone and carotid artery calcification in these patients. They concluded that seemingly, the presence of pulp stone cannot be a diagnostic marker for atherosclerotic carotid [17]. Sener et al. investigated the prevalence and predisposing factors of pulp chamber calcifications in Turkey [18]. They studied the correlation among pulp chamber calcification, dental status, gender, age, and cardiovascular disease. The periapical and bite-wing radiographs of 15,326 teeth of 536 dental patients (270 males and 266 females) aged 13 - 65 years were studied. Pulp chamber calcification was identified in 204 (38\%) of the patients. 747 (4.8\%) teeth out of 15,326 showed pulp chamber calcification. Gender and dental status correlated with pulp stone. The highest degree of prevalence of pulp stone was observedin decayed teeth, restored teeth, and restoration with caries showing that pulp stone may be reaction to long-term irritation. Anyhow, much research is still needed to report any correlation between pulp stone prevalence and various dental or clinical conditions. The present study concluded that there is the need for new studies with large numbers of subjects and different populations to find a correlation between pulp stone and cardiovascular diseases. Satheeshkumar et al. [19]. Investigated idiopathic pulpal calcifications in Southern India. A total of 227 patients aged 15 - 70 years were studied. The panoramic digital radiographs were interpreted for the presence or absence of pulp stones. The prevalence of pulp stone was more in the molar teeth of both jaws. They concluded that the idiopathic pulpal calcifications are the radiographic findings of pulp tissue. It may also be considered as a marker and index of the involved diseases. In the study above, it was concluded that the dentists' attention to dental radiographs for more accurate studies and referring the relatively older patients with high degrees of pulpal stones to cardiologists are very important steps.

\section{Conclusion}

It is recommendable that the dentists, who order panoramic radiographs for the treatment of patients, exactly evaluate teeth for the presence of pulp stones. In the case of the presence of several pulp stones, they should refer the patients to cardiologists for consultation. New research with larger samples is advisable. Specifically, a greater number of male subjects to obtain more accurate result are recommended. 


\section{Acknowledgements}

This study funded by shahid Sadoughi University of medical sciences and Iran health faculty. We thank our patients and cardiac clinic personal for good cooperation with this study.

\section{Conflict of Interest}

There is no conflict of interest.

\section{References}

[1] Sadr Bafqi, S.M., Salari, M., Rafiee, M., Nemayandeh, S.M., Abdoli, A.M., Karimi, M., et al. (2006) Prevalence and Criteria of Metabolic Syndrome in an Urban Population: Yazd Healthy Heart Project. Tehran University Medical Journal, 64, 90-96.

[2] White, S. and Pharoah, M. (2009) Oral Radiology Principles and Interpretation. Mosby, St. Louis.

[3] Ninomiya, M., Ohishi, M., Kido, J., Ohsaki, Y. and Nagata, T. (2001) Immunohistochemical Localization of Osteopontin in Human Pulp Stone. JOE, 27, 269-272.

[4] Harrison, T.R. (2002) Harrison's Principles of Internal Medicine. McGraw-Hill, Philadelphia.

[5] Little, J., Falace, D., Miller, C. and Rhodus, N. (2002) Dental Management of the Medically Compromised Patient. Mosby, London.

[6] Kajander, E.O., Ciftcioglu, N., Aho, K. and Garcia Cuerpo, E. (2003) Characteristics of Nanobacteria and Their Possible Role in Stone Formation. Urological Research, 31, 47-54.

[7] Allison, M.A., Tiefenbrun, J., Langer, R.D. and Wright, C.M. (2005) Atherosclerotic Calcification and Intimal Medial Thickness of the Carotid Arteries. International Journal of Cardiology, 103, 98-104. http://dx.doi.org/10.1016/j.ijcard.2004.12.039

[8] Nayak, M., Kumar, J. and Krishna Prasad, L. (2010) A Radiographic Correlation between Systemic Disorders and Pulp Stones. Indian Journal of Dental Research, 21, 369-373. http://dx.doi.org/10.4103/0970-9290.70806

[9] Edds, A.C., Walden, J.E., Scheetz, L.J., Drisko, G.L. and Eleazer, P.D. (2005) Pilot Study of Correlation of Pulp Stones with Cardiovascular Disease. JOE, 31, 504-506.

[10] Maranhao de Moura, A.A. and de Paiva, J.G. (1987) Pulpalcalcifications in Patients with Coronary Atherosclerosis. Endodontics \& Dental Traumatology, 3, 307-309. http://dx.doi.org/10.1111/j.1600-9657.1987.tb00640.x

[11] Khojastepour, L., Bronoosh, P., Khosropanah, S. and Rahimi, R. (2013) Can Dental Pulp Calcification Predict the Risk of Ischemic Cardiovascular Disease? Journal of Dentistry, 10, 456-460.

[12] Pornprasertsuk-Damrongsri, S. and Thanakun, S. (2006) Carotid Artery Calcification Detected on Panoramic Radiographs in a Group of Thai Population. Oral Surgery, Oral Medicine, Oral Pathology, Oral Radiology, and Endodontology, 101, 110-115. http://dx.doi.org/10.1016/j.tripleo.2005.04.002

[13] Zeng, J.F., Zhang, W., Jiang, H.W. and Ling, J.Q. (2006) Isolation, Cultivation and Initial Identification of Nanobacteria from Dental Pulp Stone. Chinese Journal of Stomatology, 41, 498-501.

[14] Tamse, A., Kaffe, I., Littner, M.M. and Shani, R. (1982) Statistical Evaluation of Radiologic Survey of Pulp Stones. Journal of Endodontics, 8, 455-458. http://dx.doi.org/10.1016/S0099-2399(82)80150-7

[15] Ezoddini-Ardakani, F., Namayandeh, S.M., Sadr-Bafghi, S.M., Fatehi, F., Mohammadi, Z., Shahrabi-Farahani, S., Hedayati, A.S. and Rahmani-Baghmalek, M.J. (2011) Association of Pulp Stones with Coronary Artery Stenosis. Community Dent Health, 28, 305-307.

[16] Horsley, S.H., Beckstrom, B., Clark, S.J., Scheetz, J.P., Khan, Z. and Farman, A.G. (2009) Prevalence of Carotid and Pulp Calcifications: A Correlation Using Digital Panoramic Radiographs. International Journal of Computer Assisted Radiology and Surgery, 4, 169-173. http://dx.doi.org/10.1007/s11548-008-0277-7

[17] Kansu, O., Ozbek, M., Avcu, N., Aslan, U., Kansu, H. and Genctoy, G. (2009) Can Dental Pulp Calcification Serve as a Diagnostic Marker for Carotic Artery Calcification in Patients with Renal Diseases? Dentomaxillofacial Radiology, 38, 542-545. http://dx.doi.org/10.1259/dmfr/13231798

[18] Sener, S., Cobankara, F.K. and Akgunlu, F. (2009) Calcifications of the Pulp Chamber: Prevalence and Implicated Factors. Clinical Oral Investigations, 13, 209-215. http://dx.doi.org/10.1007/s00784-008-0212-x

[19] Satheeshkumar, P.S., Mohan, M.P., Saji, S., Sadanandan, S. and George, G. (2013) Idiopathic Dental Pulp Calcifications in a Tertiary Care Setting in South India. Journal of Conservative Dentistry, 16, 50-55.

http://dx.doi.org/10.4103/0972-0707.105299 


\section{List of Abbreviations}

CVD: Cardiovascular Disorders

IHD: Ischemic Heart Disease

GBD: Global Burden of Disease

IGTT: Impaired Glucose Tolerance Test

ECG (EKG): Electrocardiography

IMT: Intimal Medial Thickness

CAC: Carotid Artery Calcification

ICD: Ischemic Cardiovascular Disorder

ABI: Ankle Brachial Index

\section{Appendix: Pulp Stone and Cardiovascular Disease Form}

A) Demographic data:

\section{Patient's code:}

Date:

Patient's name:

Father's name:

Birth date:

Gender: male female:

Physician's name:

Job:

Office worker

industrial worker

farmer

student retired

housekeeper

others

Tel number:

Address:

Please define the teeth and teeth with pulp stone by $\times$ Tooth with pulp stone

\section{Present tooth}

\section{dentist}

B) Medical History \& PHP:

Symptom:

Vital Sign:

Angina

Dizziness
PR:

Functional Class

Weakness
BP:

Syncope $\square$

Dyspnea

PND

Other:

Present Clinical Situation:

Symptom Free

Stable Angina

Class:

Previous MI

Unstable Angina

Class:
Previous CAGB

STEMI
Previous PTCA

NSTEMI

HF 
C) Drug History:

\begin{tabular}{|c|c|c|c|c|c|c|}
\hline \multirow{8}{*}{$\begin{array}{l}\text { Drug } \\
\text { Class }\end{array}$} & Anti hypertensive agents & \multirow[t]{2}{*}{ BBs $\square$} & \multirow[t]{2}{*}{ CCBs $\square$} & \multirow[t]{2}{*}{ ACEIs $\square$} & \multirow[t]{2}{*}{ ARBs $\square$} & \multirow[t]{2}{*}{ Diuretics $\square$} \\
\hline & Dose/24 hr & & & & & \\
\hline & Lipid lowering agents & \multirow[t]{2}{*}{ Statins $\square$} & \multirow[t]{2}{*}{ Resins $\square$} & \multirow{2}{*}{\multicolumn{2}{|c|}{ Nicotinic acid $\square$}} & \multirow[t]{2}{*}{ Fibrates $\square$} \\
\hline & Dose/24 hr & & & & & \\
\hline & Glucose lowering agents & \multirow[t]{2}{*}{ Sulfonil urea $\square$} & \multirow[t]{2}{*}{ Acarbose $\square$} & \multirow[t]{2}{*}{ Bigua $\square$} & \multirow[t]{2}{*}{ Insulin $\square$} & \multirow[t]{2}{*}{$\begin{array}{l}\text { NPH } \square \\
\text { Regular } \square\end{array}$} \\
\hline & Dose $/ 24 \mathrm{hr}$ & & & & & \\
\hline & Anti thrombotic agent & \multirow[t]{2}{*}{ Aspirin $\square$} & \multirow[t]{2}{*}{ Ticlopidin $\square$} & \multirow{2}{*}{\multicolumn{2}{|c|}{ Clopidogrel $\square$}} & \multirow[t]{2}{*}{ Warfarin $\square$} \\
\hline & Dose/24 hr & & & & & \\
\hline
\end{tabular}

D) Lab Data:

Date:

\begin{tabular}{|c|c|c|c|c|c|c|c|c|c|}
\hline FBS & TG & Chol & HDL & LDL & ALT & AST & ESR & CRP & WBC \\
\hline Hb & & Hct & & PLT & & BUN & & Crt & \\
\hline
\end{tabular}

E) Risk Factors History:

\begin{tabular}{cccc}
\hline FH $\square$ & Cigarette $\square$ & HTN $\square$ & DLP $\square$ \\
\hline $\begin{array}{c}\text { (Father/Brother }<55 \text { y or } \\
\text { mother/sister }<65)\end{array}$ & Pack/Year: & Dur: & Dur: \\
\hline
\end{tabular}

F) Ankle Brachial Index:

Date:

Systolic BP

Right hand:

Right T.P. :

ABI:

Left hand:

Left T.P. :

$\mathrm{ABI}$ :

G) Rose Questionnaire:

1. Do you have a history of chest pain:
a) Yes
b) $\mathrm{NO}$

2. If Yes, When do you have chest pain:
a) during climbing stairs or running
b) during walking

c) at rest

d) at other occasions

3. When the pain begins during your activities, what do you do?
a) reducing speed or interrupting the activity
b) continuing the activity

4. If you interrupt your activity, what does happen?
a) pain will disappear
b) pain does not change

5. How long does it take for pain to disappear?
a) less than 10 minutes
b) more than 10 minutes
6. Define the location of pain:
a) sternum (superior, middle)
b) sternum (inferior)
c) anterior and left-side of chest
d) left arm

e) other locations (explain)

7. Do you have a history of chest pain lasting for at least half an hour?
a) yes
b) no 
The presence of five first items is considered as definite angina (Rose +).

The presence of only items 1 and 5 is considered as possible angina.

The presence of the last item is considered as possible MI.

H) ECG Data:

\begin{tabular}{|c|c|c|c|c|c|c|c|}
\hline Rate & Rhythm & Axis & ST change & T wave & SVA & VA & Q wave \\
\hline \multicolumn{2}{|c|}{ Results } & & Normal $\square$ & ischemic hea & e $\square$ & Other abno & \\
\hline
\end{tabular}

I) Stress Test before Angiography:

Result:

\begin{tabular}{|c|c|c|c|c|c|}
\hline Protocol & Mets & Minutes & \multicolumn{3}{|c|}{ Result } \\
\hline & & & Positive $\square$ & Negative $\square$ & Other: \\
\hline \multirow{7}{*}{$\begin{array}{l}\text { Recovery Time : } \\
\text { Hypotension } \\
\text { Arrhythmia } \\
\text { Hypertension } \\
\text { Chest pain } \\
\text { ECG changes }\end{array}$} & Stage & HR & $\mathbf{B} / \mathbf{P}$ & ST changes & Exercise test off due to : \\
\hline & I & & & & Weakness $\square$ \\
\hline & II & & & & Hypotension $\square$ \\
\hline & II & & & & Arrhythmia \\
\hline & III & & & & Hypertension $\square$ \\
\hline & IV & & & & Chest pain $\square$ \\
\hline & V & & & & \\
\hline
\end{tabular}

